Research Article

\title{
Effectiveness of Conservative Treatment without Early Colonoscopy in Patients with Colonic Diverticular Hemorrhage
}

\author{
Hirosato Doi (D), Keita Sasajima (D), Masanori Takahashi, Taira Sato, Iichirou Ootsu, \\ and Ryo Chinzei
}

Department of Digestive Internal Medicine, Saitama Red Cross Hospital, Saitama, Japan

Correspondence should be addressed to Keita Sasajima; qqfa98m9@mist.ocn.ne.jp

Received 10 September 2019; Revised 2 December 2019; Accepted 17 December 2019; Published 14 January 2020

Academic Editor: Toshio Uraoka

Copyright ( $) 2020$ Hirosato Doi et al. This is an open access article distributed under the Creative Commons Attribution License, which permits unrestricted use, distribution, and reproduction in any medium, provided the original work is properly cited.

\begin{abstract}
Aim. This study was aimed to clarify the effectiveness of conservative treatment without performing early colonoscopy and the indications for early colonoscopy in patients with colonic diverticular hemorrhage. Methods. This retrospective study included 142 participants who were urgently hospitalized due to bloody stools and were diagnosed with colonic diverticular hemorrhage between April 2012 and December 2016. At the time of hospital visit, only when both shock based on vital signs and intestinal extravasation on abdominal contrast-enhanced computed tomography were observed, early colonoscopy was performed within 24 hours after hospitalization. However, in other cases, patients were conservatively treated without undergoing early colonoscopy. In cases of initial treatment failure in patients with shock, interventional radiology (IVR) was performed without undergoing early colonoscopy. Results. Conservative treatment was performed in 137 (96.5\%) patients, and spontaneous hemostasis was achieved in all patients. By contrast, urgent hemostasis was performed in five (3.5\%) patients; three and two attained successful hemostasis via early colonoscopy and IVR, respectively. There were no significant differences between two groups in terms of early rebleeding $(7.3 \%$ vs. $0 \%, P=0.690)$ and recurrent bleeding $(22.7 \%$ vs. $20.0 \%, P=0.685)$. The factors associated with the cumulative recurrent bleeding rates were a previous history of colonic diverticular hemorrhage (hazard ratio 5.63, 95\% confidence interval 2.68-12.0, $P<0.0001$ ) and oral administration of thienopyridine derivative (hazard ratio 3.05 , 95\% confidence interval 1.23-7.53, $P=0.016$ ). Conclusions. In this series, conservative treatment without early colonoscopy was successful in patients with colonic diverticular hemorrhage.
\end{abstract}

\section{Introduction}

In Japan, the number of patients with colonic diverticulum has been increasing due to aging and the westernization of dietary habits, and the frequency is reportedly about one in four people $[1,2]$. In addition, along with the elevated number of patients who are taking antithrombotic drugs, the prevalence of colonic diverticular hemorrhage is increasing annually, and this condition is the most frequent cause of lower gastrointestinal bleeding, accounting for approximately $25 \%$ of the total cases [3-5].

In Japanese guidelines for colonic diverticular hemorrhage, it is proposed that early colonoscopy would be preferably performed within 24 hours of hospital visit to identify the stigma of recent hemorrhage (SRH) [6], and several institutions have actively performed early colonoscopy. In addition, some new endoscopic techniques, which might contribute to increase in successful hemostasis and decrease in rebleeding, have been recently reported, such as endoscopic band ligation, endoscopic detachable snare ligation, and over-the-scope clip (OTSC ${ }^{\circledR}$, Ovesco Endoscopy, Tübingen, Germany) [7-9]. However, it has been also reported that colonic diverticular hemorrhage generally stopped spontaneously, approximately $76 \%-91 \%$ of patients $[10,11]$. Moreover, the rate of SRH identification with early colonoscopy has been still low, only $15 \%-42 \%$ [12-16], due 
to spontaneous hemostasis during colonoscopy and multiple diverticulum in several cases, which would result in ineffective intervention for colonic diverticular hemorrhage.

On these backgrounds, we performed follow-up assessments on the use of conservative treatment alone without performing early colonoscopy, except for cases with two signs of sustained bleeding: shock based on vital signs and extravasation on contrast-enhanced computed tomography (CECT). The aim of this study was to clarify the effectiveness of conservative treatment for colonic diverticular hemorrhage with respect to primary hemostasis and rebleeding.

\section{Methods}

2.1. Patients. This study included 142 consecutive patients who met the following criteria: (i) patients with bloody stools who visited as outpatients and were urgently hospitalized between April 2012 and December 2016; (ii) colonic diverticula were observed on colonoscopy or CECT; and (iii) other bleeding sources were ruled out via upper and lower gastrointestinal tract endoscopy or abdominal CECT. This study was approved by the institutional ethics review board at our hospital, and all patients gave informed consent about risks and benefits of their treatment depending on physical condition.

2.2. Flowchart of the Treatment Options. The treatment options are shown in Figure 1. If shock (systolic blood pressure $<90 \mathrm{mmHg}$ ) was observed at the time of hospital visit, we first performed fluid administration such as rapid infusion and blood transfusion to stabilize the general condition of the patient. All patients were then subjected to abdominal CECT to assess for extravasation within the bowel lumen, unless renal dysfunction was indicated based on the blood test results (estimated glomerular filtration rate $>30 \mathrm{~mL} / \mathrm{min} / 1.73 \mathrm{~m}^{2}$ ). Whenever both shock and extravasation were observed at the time of hospital visit, early colonoscopy was definitely performed within 24 hours after admission. Additionally, if SRH was identified on early colonoscopy, hemostasis was firstly performed only by a clipping method. By contrast, if SRH was not identified due to spontaneous hemostasis during colonoscopy, follow-up observation was performed after the examination. In case of difficulty in recovering from shock or achieving endoscopic hemostasis, interventional radiology (IVR) was performed to stop bleeding by arterial embolization. Meanwhile, if vital signs were stable at the time of hospital visit or extravasation was not detected on CECT, conservative treatment was conducted without performing early colonoscopy. In these cases, elective colonoscopy was performed within 2 weeks after spontaneous hemostasis. In cases wherein CECT could not be performed due to renal dysfunction or allergy from the contrast agent, indications for early colonoscopy were determined based solely on the abovementioned guidelines on vital signs.

With regard to the indications of blood transfusion, patients with a serum hemoglobin $(\mathrm{Hb})$ level $<7 \mathrm{~g} / \mathrm{dL}$ are considered for blood transfusion. However, in some patients with severe comorbidities or general conditions, such as shock, blood transfusion is considered even if the $\mathrm{Hb}$ level is less than $9 \mathrm{~g} / \mathrm{dL}$. Food intake was started if bloody stools had not been observed for $>24$ hours. The meals were gradually solidified each day and patients were discharged with a regular diet. Antithrombotic drugs were generally discontinued in patients with shock and immediately restarted after hemostasis, while antithrombotic drugs were not discontinued with stable vital signs. Signs of rebleeding were defined as the presence of fresh bloody stool along with low blood pressure (systolic blood pressure $<90 \mathrm{mmHg}$ ) or a decrease in the $\mathrm{Hb}$ level of $\geq 2.0 \mathrm{~g} / \mathrm{dL}$. In case of rebleeding, colonoscopy was considered when both shock and extravasation on CECT were observed or continuous intermittent rebleeding was observed for $\geq 2$ days. Early rebleeding was defined as rebleeding within 30 days after hospitalization, and recurrent bleeding as massive hematochezia more than 30 days after initial hospitalization, which resulted in second hospitalization. Presence or absence of recurrent bleeding was evaluated in all patients until February 2018 according to the following ways: (i) in case of outpatients in our hospital, it was evaluated at the day of onset or recent visit; (ii) in case of patients who were not followed in our hospital, it was evaluated by telephone call with questionnaire survey, which provided recent history about presence or absence of hospitalization due to hematochezia.

2.3. Routine Setting of Colonoscopy. Colonoscopy, including early colonoscopy, is performed after preparation with $2 \mathrm{~L}$ of polyethylene glycol (PEG). If patients have difficulty ingesting PEG due to poor general condition, either highpressure enema or no preparation was carried out before colonoscopy. Carbon dioxide insufflation was used in all patients to reduce abdominal discomfort except for those with chronic obstructive pulmonary disease. PCF-Q260AZI (Olympus, Tokyo, Japan), which has a water jet system, was used for early colonoscopy with a cap attachment and PCFQ260AZI or CF-H260AZI (Olympus, Tokyo, Japan) for elective colonoscopy.

2.4. Clipping. If SRH was identified, hemostasis was firstly performed only by a clipping method for all patients. Clips (HX-610-135; Olympus, Tokyo, Japan) were placed directly on the visible vessel or stigmata if possible. When direct placement was difficult because of a diverticular dome location, massive hemorrhage, or small diverticular orifice, indirect placement was performed with multiple clips in a zipper fashion $[17,18]$.

2.5. Interventional Radiology. IVR was performed in the femoral artery using 4-Fr Shepherd hook catheter, and nonionic contrast medium was injected in the superior mesenteric artery $(5 \mathrm{~mL} / \mathrm{s})$ and inferior mesenteric artery $(3 \mathrm{~mL} / \mathrm{s})$ to identify the bleeding sites. Once the bleeding sites were identified, a microcatheter was carefully advanced 


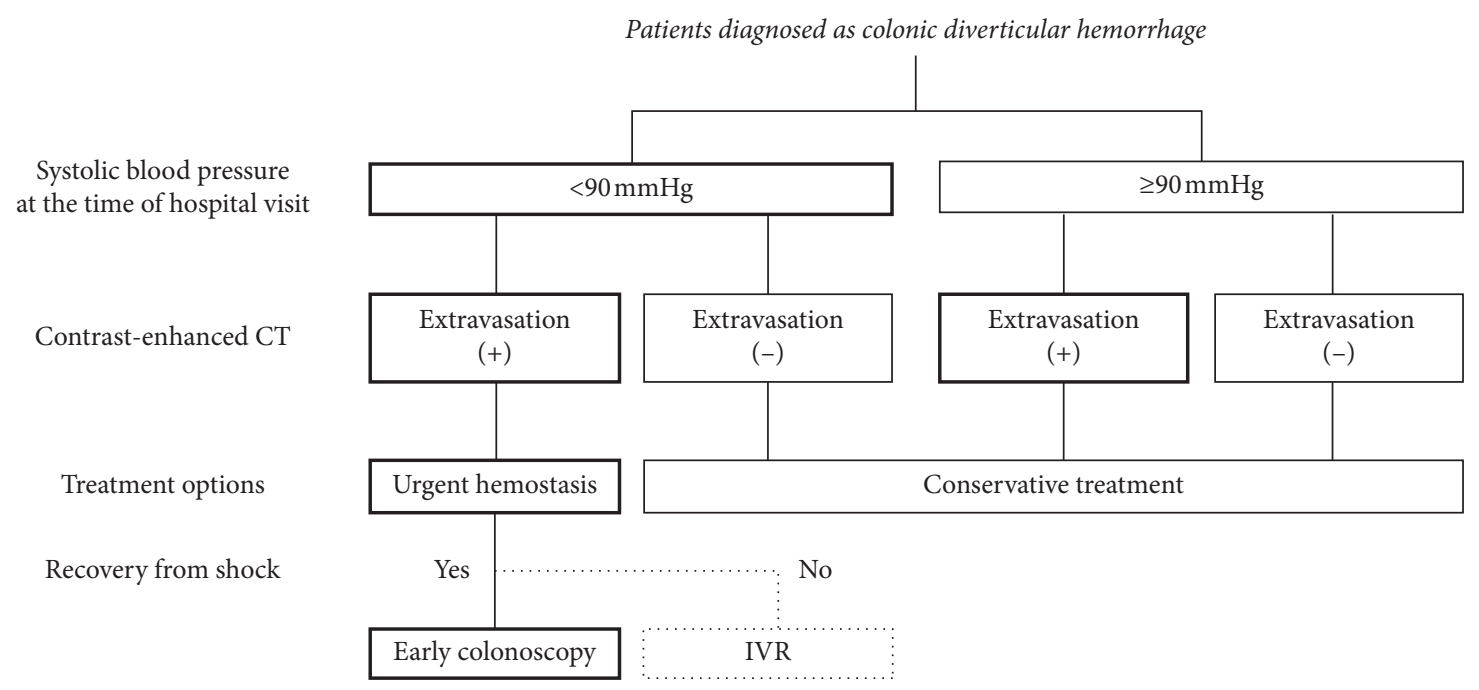

FIGURE 1: Flowchart of the treatment options for colonic diverticular hemorrhage. CT, computed tomography; IVR, interventional radiology. In case with both shock and extravasation on contrastenhanced computed tomography, early colonoscopy was performed. However, in case of uncontrolled hemorrhagic shock after initial treatment with fluid resuscitation, interventional radiology was considered for hemostasis. In other cases, conservative treatment with fasting and fluid administration was conducted.

to the bleeding site, and arterial embolization was performed using coils.

2.6. Statistical Analysis. Mean \pm standard deviation or percentage was used for all data. Categorical data were compared using the chi-square test or Fisher's exact test, and continuous data were compared using the Wilcoxon rank sum test. The relationship between necessity of early colonoscopy and backgrounds was examined using Fisher's exact test; multivariate logistic regression analyses were unsuitable and not used due to a small number of cases in the urgent hemostasis group. Kaplan-Meier method and the log-rank test were used in the time-to-event analysis in patients with recurrent bleeding, and the Cox proportional hazard model was performed to examine the factors affecting recurrent bleeding. In the statistical analysis, JMP (version 13; SAS Institute Inc., USA) was used, and a $P$-value $<0.05$ was considered statistically significant.

\section{Results}

3.1. Initial Hemostasis. Characteristics of the patients are shown in Table 1 . Among the participants, 88 were males and 54 were females with an average age of $71.7 \pm 12.7$ (range: 34-94) years, and hemorrhagic shock was observed in $11.3 \%$ of the patients. The results are shown in Table 2. Of the 142 patients with colonic diverticular hemorrhage, 137 (96.5\%) received conservative treatment, and only five (3.5\%) underwent urgent hemostasis. In the conservative treatment group, $11(8.0 \%)$ patients presented with shock at the time of hospital visit. However, spontaneous hemostasis was achieved in all patients by fasting and gut rest, and none of the patients required hemostasis by elective colonoscopy. Only one $(0.7 \%)$ patient in the conservative treatment group died due to bacterial pneumonia, and not from hemorrhagic shock. Blood transfusion was performed in $60(43.8 \%)$ patients, and the mean $\mathrm{Hb}$ level at the time of blood transfusion was $7.2 \pm 1.3 \mathrm{~g} / \mathrm{dL}$, and the mean transfusion volume was $2.7 \pm 4.0$ units.

In contrast, in the urgent hemostasis group, early colonoscopy was performed in four of five patients and $\mathrm{SRH}$ was identified in three patients, resulting in successful hemostasis by clipping. Clips could be placed directly on SRH in two of the three patients and indirectly in one patient due to small diverticular orifice. In the other one of four patients, SRH could not be identified due to severe bleeding, and one patient could not receive early colonoscopy due to difficulty in recovering from shock. They received IVR, resulting in successful hemostasis. Consequently, hemostasis was achieved successfully in all patients and no patients died with hemorrhagic shock. In the urgent hemostasis group, bleeding sites were all located in the right colon and three of five patients took oral antithrombotic drugs.

The association between the necessity of early colonoscopy and the risk factors were examined (Table 3), and shock based on vital sings and extravasation on CECT at the time of hospital visit were only risk factors of early colonoscopy.

\subsection{Incidence of Rebleeding after Treatment}

3.2.1. Early Rebleeding. Early rebleeding was not observed in all the patients in the urgent hemostasis group, whereas it was observed in $7.3 \%$ [95\% confidence interval (CI): 4.0-12.9] of the patients in the conservative treatment group. There were no significant differences between the two groups. In addition, all the patients with early rebleeding achieved spontaneous hemostasis with conservative treatment. There was no significant relationship between early rebleeding and clinical backgrounds.

3.2.2. Recurrent Bleeding. In the conservative treatment group, recurrent bleeding was observed in 29 of 128 patients $(22.7 \%$; 
95\% CI: 16.3-30.6) during the observation periods of $31.3 \pm 18.6$ (14-68) months, excluding those with duplicated cases. It occurred 2-38 $(12.4 \pm 11.3)$ months after initial conservative treatment. The cumulative recurrent bleeding rates were $12.1 \%, 22.1 \%$, and $31.6 \%$ in 1,3 , and 5 years, respectively (Figure 2). Meanwhile, recurrent bleeding in the urgent hemostasis group was observed in one of 5 patients (20.0\%, 18 months after hemostasis) during observation periods of $29.5 \pm 13.5(18-51)$ months. In this case, as recurrent life-threatening hemorrhage occurred at the same site as previous treatment, elective surgical treatment was performed. There were no significant differences between the two groups in terms of recurrent bleeding rates.

Univariate analysis using the log-rank test showed that the recurrent bleeding rate was significantly higher in patients with a previous history of diverticular hemorrhage and oral administration of low-dose aspirin and thienopyridine derivative (Table 4). The cumulative recurrent bleeding rates in the group without a previous history of diverticular hemorrhage were $6.1 \%, 10.5 \%$, and $12.0 \%$ in 1,2 , and 3 years, whereas those with a history of diverticular hemorrhage were $26.9 \%, 47.0 \%$, and $58.7 \%$ in 1,2 , and 3 years, respectively $(P<0.0001)$. The cumulative recurrent bleeding rates in the non-antithrombotic drug group were $6.5 \%$, $13.5 \%$, and $17.9 \%$ in 1,2 , and 3 years, whereas those in the low-dose aspirin group were $18.8 \%, 28.4 \%$, and $39.3 \%$ in 1,2 , and 3 years, respectively $(P=0.0216)$, and those in the thienopyridine derivative group were $21.4 \%, 43.8 \%$, and $53.1 \%$ in 1,2 , and 3 years, respectively $(P=0.0021)$. Furthermore, in only patients who received dual antiplatelet therapy (DAPT), recurrent bleeding occurred in 5 of 9 patients (55.6\%), and the cumulative recurrent bleeding rate was also significantly higher at $37.5 \%$ after 1 year and $81.3 \%$ after 2 years compared to the non-antithrombotic drug group $(P<0.0001)$.

Multivariate analyses using Cox proportional hazard model showed that patients with a previous history of diverticular hemorrhage (hazard ratio 5.63, 95\% CI 2.68-12.0, $P<0.0001)$ or oral administration of thienopyridine derivative (hazard ratio $3.05,95 \%$ CI $1.23-7.53, P=0.016$ ) were significantly associated with recurrent bleeding.

3.3. Length of Hospital Stay and Cost. The length of fasting did not significantly differ between the conservative treatment and urgent hemostasis groups $(4.1 \pm 1.7$ vs. $4.8 \pm 1.9$ days, $P=0.3418$ ). The hospitalization period of the conservative treatment group was significantly shorter than that of the urgent hemostasis group $(11.3 \pm 5.9$ vs. $19.2 \pm 21.4$ days, $P=0.0395)$. The hospitalization cost was significantly higher in the urgent hemostasis group compared to the conservative treatment group $(\$ 8237.28 \pm 3211.87$ vs. $\$ 4270.68 \pm 2452.30, P=0.0001)$, even in the case except for the cost of IVR $(\$ 7169.06 \pm 2168.09, P=0.0468)$.

\section{Discussion}

In Japan, colonic diverticular hemorrhage is increasingly common with aging population and westernization of
TABLE 1: Characteristics of patients.

\begin{tabular}{lc}
\hline Patients $(n=142)$ & \\
\hline Age (years, mean \pm SD) & $71.7 \pm 12.7$ \\
Sex (male/female) & $(34-94)$ \\
Localization of diverticulum & $88 / 54$ \\
$\quad$ Right-sided & $38(26.8 \%)$ \\
Left-sided & $14(9.9 \%)$ \\
Bilateral & $90(63.4 \%)$ \\
Previous history of diverticular hemorrhage & $38(26.8 \%)$ \\
Patient on dialysis & $11(7.7 \%)$ \\
Patient with cirrhosis & $3(2.1 \%)$ \\
Medications & \\
Antithrombotic drugs (total) & $56(39.4 \%)$ \\
Aspirin & $37(26.1 \%)$ \\
Thienopyridine derivative & $17(12.0 \%)$ \\
Anticoagulants & $23(16.2 \%)$ \\
Dual antiplatelet therapy & $10(7.0 \%)$ \\
NSAIDs & $14(9.6 \%)$ \\
Systolic blood pressure $(\mathrm{mmHg}, \mathrm{mean} \pm \mathrm{SD})$ & $127.2 \pm 30.7$ \\
Hemorrhagic shock & $16(11.3 \%)$ \\
Heart rate (bpm, mean $\pm \mathrm{SD})$ & $86.6 \pm 20.5$ \\
Loss of consciousness & $17(12.0 \%)$ \\
Extravasation on CECT & \\
Laboratory data & $18(18.4 \%)$ \\
Hemoglobin level $(\mathrm{g} / \mathrm{dL}, \mathrm{mean} \pm \mathrm{SD})$ & \\
White blood cell count $\left(\times 10^{3} / \mathrm{mm}^{3}\right.$, & $10.5 \pm 2.5$ \\
mean \pm SD) & $8.1 \pm 2.9$ \\
Platelet count $\left(\times 10^{4} / \mathrm{mm}^{3}, \mathrm{mean} \pm \mathrm{SD}\right)$ & $20.4 \pm 6.1$ \\
UN/Cre ratio (mean $\pm \mathrm{SD})$ & $23.0 \pm 10.4$ \\
Albumin level $(\mathrm{g} / \mathrm{dL}, \mathrm{mean} \pm \mathrm{SD})$ & $3.5 \pm 0.5$ \\
PT-INR (mean $\pm \mathrm{SD})$ & $1.2 \pm 0.7$ \\
\hline
\end{tabular}

SD, standard deviation; NSAIDs, nonsteroidal anti-inflammatory drugs; CECT, contrast-enhanced computed tomography; UN/Cre, urea nitrogen/ creatinine; PT-INR, international normalized ratio of prothrombin time. ${ }^{*} \mathrm{CECT}$ was performed in ninety-eight patients.

dietary habits, and valuable treatment options should be fully discussed for the management of colonic diverticular hemorrhage. Currently, Japanese guidelines proposed early colonoscopy as the triage tool for patients with sustained bleeding although it remains controversial whether early colonoscopy would contribute to increase in identification of SRH or prevention of rebleeding [6]. On the contrary, our results in a real clinical setting suggested that colonic diverticular hemorrhage would stop spontaneously without any interventions, i.e., with only conservative treatment, except for the cases with shock based on vital signs and extravasation on CECT. In addition, interventions of urgent hemostasis contributed little to decrease in recurrent bleeding compared to conservative treatment.

Recently, a multicenter randomized control trial demonstrated that early colonoscopy for acute lower gastrointestinal bleeding did not improve the rate of $\mathrm{SRH}$ identification and reduce rebleeding [19], and this report would strongly support the results of our study. Moreover, the trial revealed that the rate of SRH identification was $21.3 \%(10 / 47)$. However, the primary endpoint of our study was a rate of successful hemostasis spontaneously by conservative treatment which was quite high of $96.5 \%$. We hypothesized that most of SRH such as visible vessel and 
TABLE 2: Treatment outcomes.

\begin{tabular}{|c|c|c|c|}
\hline & Conservative treatment $(n=137)$ & Urgent hemostasis $(n=5)$ & $P$-value \\
\hline Hemostasis & $137(100 \%)$ & $5(100 \%)^{*}$ & \\
\hline \multicolumn{4}{|l|}{ Mortality } \\
\hline Bleeding & $0(0 \%)$ & $0(0 \%)$ & 0.978 \\
\hline Others & $1(0.7 \%)$ & $0(0 \%)$ & \\
\hline Blood transfusion & $60(43.8 \%)$ & $5(100 \%)$ & 0.003 \\
\hline Units of blood (mean \pm SD) & $2.7 \pm 4.0$ & $10.0 \pm 3.3$ & 0.0002 \\
\hline $\mathrm{Hb}$ level during transfusion $(\mathrm{g} / \mathrm{dL}$, mean $\pm \mathrm{SD})$ & $7.2 \pm 1.3$ & $7.0 \pm 1.5$ & \\
\hline Hemorrhagic shock (systolic $\mathrm{BP}<90 \mathrm{mmHg}$ ) & $11(8.0 \%)$ & $5(100 \%)$ & $<0.0001$ \\
\hline Periods of fasting (days, mean \pm SD) & $4.1 \pm 1.7(1-9)$ & $4.8 \pm 1.9(4-7)$ & 0.342 \\
\hline Length of hospitalization (days, mean \pm SD) & $11.3 \pm 5.9(3-34)$ & $19.2 \pm 21.4(9-30)$ & 0.040 \\
\hline \multirow{2}{*}{ Hospitalization costs $(\mathrm{USD}$, mean $\pm \mathrm{SD})$} & \multirow{2}{*}{$4270.68 \pm 2452.30$} & $8237.28 \pm 3211.87$ & 0.0001 \\
\hline & & $7169.06 \pm 2168.09^{* *}$ & 0.047 \\
\hline Early rebleeding & $10(7.3 \%)^{* * *}$ & $0(0 \%)$ & 0.690 \\
\hline Recurrent bleeding & $29(22.7 \%)$ & $1(20 \%)$ & 0.685 \\
\hline
\end{tabular}

SD, standard deviation; Hb, hemoglobin; BP, blood pressure; USD, the United States dollar. *Three of five patients underwent endoscopic hemostasis, and the other two patients underwent transcatheter arterial embolization, resulting in successful hemostasis in all patients. ${ }^{* *}$ Except the two patients who received interventional radiology treatment, ${ }^{* * *}$ all patients achieved spontaneous hemostasis after early rebleeding.

TABLE 3: Factors associated with urgent hemostasis.

\begin{tabular}{|c|c|c|c|}
\hline & Conservative treatment $(n=137)$ & Urgent hemostasis $(n=5)$ & $P$-value \\
\hline Age (years, mean $\pm \mathrm{SD})$ & $71.6 \pm 12.8$ & $74.2 \pm 11.5$ & 0.652 \\
\hline Sex (male) & $84(61.3 \%)$ & $4(80.0 \%)$ & 0.650 \\
\hline Previous history of diverticular hemorrhage & $36(26.3 \%)$ & $2(40.0 \%)$ & 0.610 \\
\hline Patient on dialysis & $11(8.3 \%)$ & $0(0 \%)$ & 0.999 \\
\hline Patient with cirrhosis & $3(2.2 \%)$ & $0(0 \%)$ & 0.999 \\
\hline \multicolumn{4}{|l|}{ Medications } \\
\hline Antithrombotic (total) & $53(38.9 \%)$ & $3(60.0 \%)$ & 0.383 \\
\hline Aspirin & $35(25.5 \%)$ & $2(40.0 \%)$ & 0.605 \\
\hline Thienopyridine derivative & $16(11.7 \%)$ & $1(20.0 \%)$ & 0.477 \\
\hline Dual antiplatelet therapy & $9(6.6 \%)$ & $1(20.0 \%)$ & 0.310 \\
\hline Anticoagulants & $21(15.3 \%)$ & $2(40.0 \%)$ & 0.189 \\
\hline NSAIDs & $13(9.5 \%)$ & $1(20.0 \%)$ & 0.410 \\
\hline Systolic blood pressure $<90(\mathrm{mmHg})$ & $11(8.3 \%)$ & $5(100 \%)$ & $<0.0001$ \\
\hline Heart rate $(\mathrm{bpm}$, mean $\pm \mathrm{SD})$ & $87.0 \pm 20.4$ & $74.2 \pm 22.8$ & 0.184 \\
\hline Loss of consciousness & $17(12.4 \%)$ & $0(0 \%)$ & 0.999 \\
\hline Extravasation on CECT & $13(14.0 \%)^{*}$ & $5(100 \%)$ & 0.0001 \\
\hline $\begin{array}{l}\text { Localization of diverticulum (right-sided/left-sided/ } \\
\text { bilateral) }\end{array}$ & $34 / 14 / 89$ & $4 / 0 / 1$ & 0.004 \\
\hline \multicolumn{4}{|l|}{ Laboratory data } \\
\hline Hemoglobin level $(\mathrm{g} / \mathrm{dL}$, mean $\pm \mathrm{SD})$ & $10.5 \pm 2.5$ & $10.9 \pm 3.4$ & 0.696 \\
\hline WBC count $\left(\times 10^{3} / \mathrm{mm}^{3}\right.$, mean $\left.\pm \mathrm{SD}\right)$ & $8.1 \pm 2.9$ & $8.1 \pm 4.0$ & 0.988 \\
\hline Platelet count $\left(\times 10^{4} / \mathrm{mm}^{3}\right.$, mean $\left.\pm S D\right)$ & $20.5 \pm 6.2$ & $18.9 \pm 2.0$ & 0.584 \\
\hline $\mathrm{UN} /$ Cre ratio $($ mean $\pm \mathrm{SD})$ & $23.1 \pm 10.5$ & $19.1 \pm 3.4$ & 0.390 \\
\hline Albumin level $(\mathrm{g} / \mathrm{dL}$, mean $\pm \mathrm{SD})$ & $3.5 \pm 0.5$ & $3.2 \pm 0.5$ & 0.211 \\
\hline PT-INR $($ mean \pm SD) & $1.2 \pm 0.7$ & $1.5 \pm 0.6$ & 0.429 \\
\hline
\end{tabular}

SD, standard deviation; NSAIDs, nonsteroidal anti-inflammatory drugs; CECT, contrast-enhanced computed tomography; WBC, white blood cells; UN/Cre, urea nitrogen/creatinine; PT-INR, international normalized ratio of prothrombin time. ${ }^{*}$ CECT was performed in ninety-three patients in the conservative treatment group.

adherent clot in early colonoscopy might result in spontaneous hemostasis only with conservative treatment.

Most of diverticular hemorrhage, which is generally thought to be caused by a breakage in the vulnerable part of the vasa recta in protrusions, basically results in venous bleeding [20, 21] and would stop spontaneously without immediate hemostasis. In fact, previous reports have shown that spontaneous hemostasis occurred in $76 \%-91 \%$ of all cases $[10,11]$, and our result was also consistent with this principle. By contrast, some diverticular hemorrhage could be caused by arterial bleeding, which would require urgent hemostasis and have high risk of rebleeding even if it stops spontaneously. It should be crucial to assess whether hemorrhage was caused by arterial or venous bleeding.

In this study, the criteria for early colonoscopy were coexistence of shock at the time of hospital visit and extravasation on CECT, which were indicators of active bleeding. Although shock in patients with diverticular 


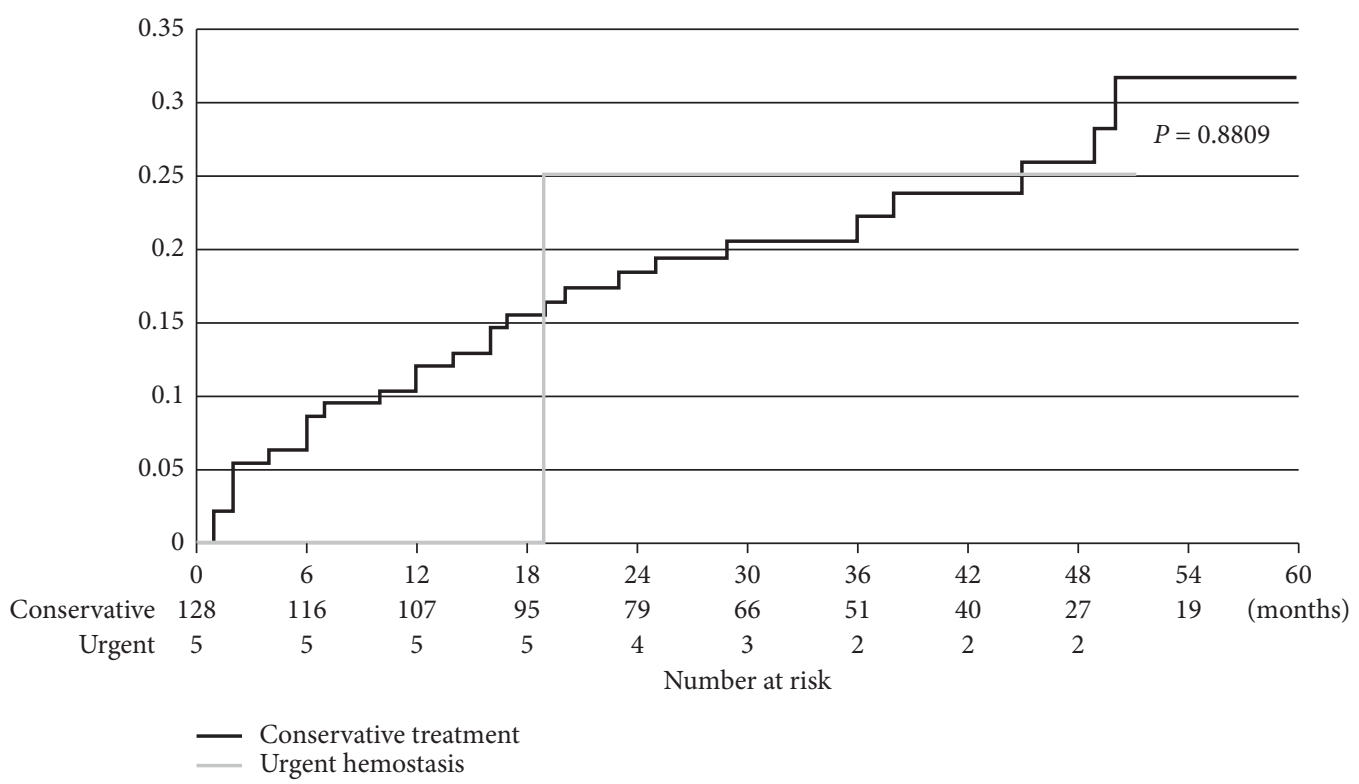

FIGURE 2: Recurrent bleeding rates after conservative treatment and urgent hemostasis. During a follow-up period of $31.2 \pm 18.4$ months, the overall recurrent bleeding rate after conservative treatment and urgent hemostasis were $22.7 \%$ and $20.0 \%$, respectively. The cumulative recurrent bleeding rate after conservative treatment was $12.1 \%$ after 1 year, $22.1 \%$ after 3 years, and $31.6 \%$ after 5 years.

hemorrhage might contribute to temporary hypovolemic shock due to arterial bleeding, there is another possibility of vagal reflex in several cases. In addition, CECT, which could identify the localization of the bleeding sites $[22,23]$ and predict the risk of developing unstable vital signs after hospitalization, can detect bleeding as small as $0.5 \mathrm{~mL} / \mathrm{min}$ $[24,25]$, which indicates that extravasation does not necessarily represent arterial bleeding. Therefore, we hypothesized that spontaneous hemostasis might be most likely achieved if only one of the two was observed, and conservative treatment could be the first choice of treatment in these cases. In fact, in these cases, spontaneous hemostasis was achieved with conservative treatment in all patients. By contrast, in case with both shock and extravasation on CECT, two of five patients suffered severe life-threatening hemorrhage which required hemostasis by IVR. Moreover, SRH could be all identified in the other three cases during early colonoscopy. Thus, we believe that the criteria of early colonoscopy used in this study would be highly likely to be appropriate, and the prospective study should be conducted with large sample size in the future.

Based on several meta-analyses, early colonoscopy for diverticular hemorrhage improved identification of SRH although it did not significantly improve mortality rate, rebleeding rate, and duration of hospital stay compared with those of elective colonoscopy cases [14, 26-28]. In addition, when performing early colonoscopy, preparation with PEG is basically indispensable in improving the rate of SRH identification $[16,29]$. The majority of patients with diverticular hemorrhage are elderly and the ingestion of PEG forces a significant load in patients with a poor general condition. Considering the fact that spontaneous hemostasis occurred in most cases, the indication for early colonoscopy should be further narrowed down. If early colonoscopy was assumed to be performed in all cases, patients would then be transferred to facilities that could offer early colonoscopy, and the load in these facilities would rapidly increase.

It has been reported that the rate of early rebleeding within 30 days was $15 \%-30 \%$ in either the endoscopic hemostasis group or the conservative treatment group $[14,15,30,31]$, and the long-term recurrent bleeding rate was $14 \%-40 \%[10,14,18,32,33]$. The early rebleeding rate in this study was favorable, i.e., approximately $7.3 \%$ in the conservative treatment group and $0 \%$ in the urgent hemostasis group. In the conservative treatment group, if rebleeding occurred, spontaneous hemostasis was again achieved by conservative treatment in all cases. When assessing risk factors for early rebleeding, neither conservative treatment nor urgent hemostasis was considered as a significant risk factor. Moreover, the severity of initial bleeding was not associated with early rebleeding. Meanwhile, in recurrent bleeding, the 5-year cumulative recurrent bleeding rate was $31.6 \%$ in the conservative treatment group, which was almost similar to the recurrent bleeding rates in institutions where hemostasis intervention was actively performed [18, 32, 34]. This result was consistent with previous reports showing that early colonoscopy did not contribute to the reduced incidence of recurrent bleeding [14]. With regard to the risk factors of recurrent bleeding, a previous history of diverticular hemorrhage as well as use of thienopyridine derivative was significantly associated with recurrent bleeding, although there was a report showing that the use of nonsteroidal anti-inflammatory drugs and antiplatelet drugs as well as high blood pressure increased the frequency of rebleeding $[16,33,35,36]$. Moreover, in the present study, patients with DAPT had an extremely high recurrent bleeding rate, which was also anticipated to increase. Further studies on high-risk groups with recurrent bleeding must be conducted. 
TABLE 4: Factors associated with recurrent bleeding after spontaneous hemostasis.

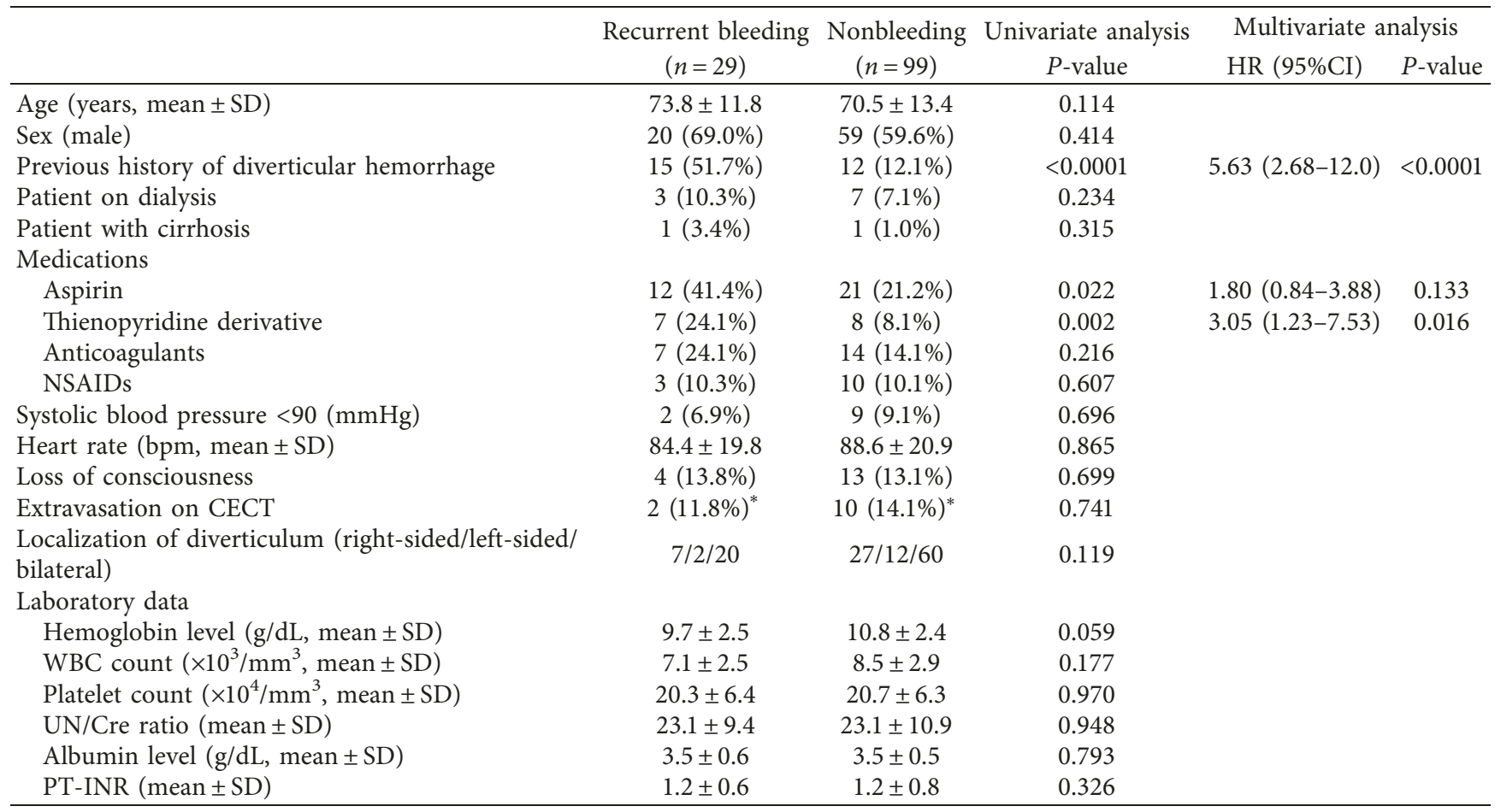

Follow-up period: $31.3 \pm 18.6$ months. HR, hazard ratio; CI, confidence interval; SD, standard deviation; NSAIDs, nonsteroidal anti-inflammatory drugs; CECT, contrast-enhanced computed tomography; WBC, white blood cells; UN/Cre, urea nitrogen/creatinine; PT-INR, international normalized ratio of prothrombin time. ${ }^{*} \mathrm{CECT}$ was performed in seventeen patients in the recurrent bleeding group and in seventy-one patients in the nonbleeding group.

The median period of hospitalization in the conservative treatment group in the present study was 10 days, which was similar to the report from an institution that performed early colonoscopy for colonic diverticular hemorrhage in Japan [37]. Because the cost for hemostasis or the additional cost for hospitalization due to serious conditions is not involved in the conservative treatment, medical expenses for hospitalization are reduced. This was confirmed in our study even though the number of patients was relatively small. Issues related to medical expenses would vary depending on the circumstances of each country; however, because spontaneous hemostasis could be achieved without any other treatments in majority of the cases, there is no doubt that conservative treatment is superior to other treatments in terms of medical costs. On the other hand, periods of fasting in the conservative treatment group seemed long (4.1 days) and length of hospitalization was longer compared to that in the RCT report (11.3 days vs. 7.6 days) although subjects were different between the two studies [19]. These facts would be attributed to our study design of retrospective study; resumption of food intake and timing of discharge were decided at the individual discretion of bedside physicians. From the viewpoint of "budgeting," a longer hospital stay can become a burden to hospital management. To solve the issue, a prospective study was conducted and is now ongoing (UMIN000028007).

This study has several strengths. First, conservative treatment without early colonoscopy would be a candidate for most patients, except for cases with both shock based on vital signs and extravasation on CECT. Second, conservative treatment without early colonoscopy would be fewer burdens on patients economically and physically compared to early colonoscopy. By contrast, our study has several limitations. First, this is a retrospective consecutive study, not a randomized controlled trial; the study was performed at a single facility, and the sample size was relatively small especially in the urgent hemostasis group. Our results should be certainly validated in a multicenter prospective study. Second, CECT was performed in all patients because it has the clinical benefits to identify bleeding sources including rectal ulcer, colorectal cancer, ischemic colitis, and so on, except for diverticular hemorrhage $[22,23]$. The identification would allow for efficient clinical management depending on each bleeding source.

\section{Conclusion}

Conservative treatment without performing early colonoscopy was a useful therapeutic option for the patients with colonic diverticular hemorrhage to achieve spontaneous hemostasis, except for those with both shock and extravasation on CECT.

\section{Data Availability}

The data used to support the findings of this study are included within the article.

\section{Conflicts of Interest}

The authors declare that there are no conflicts of interest regarding the publication of this paper. 


\section{Acknowledgments}

The authors would like to express their gratitude to Dr. Tetsuya Mizoue from the National Center for Global Health and Medicine for providing statistical advice.

\section{References}

[1] N. Yamamichi, T. Shimamoto, Y. Takahashi et al., "Trend and risk factors of diverticulosis in Japan: age, gender, and lifestyle/metabolic-related factors may cooperatively affect on the colorectal diverticula formation," PLoS One, vol. 10, no. 4, Article ID e0123688, 2015.

[2] N. Nagata, R. Niikura, T. Shimbo et al., "Alcohol and smoking affect risk of uncomplicated colonic diverticulosis in Japan," PLoS One, vol. 8, no. 12, Article ID e81137, 2013.

[3] R. Niikura, H. Yasunaga, Y. Yamaji et al., "Factors affecting inhospital mortality in patients with lower gastrointestinal tract bleeding: a retrospective study using a national database in Japan," Journal of Gastroenterology, vol. 50, no. 5, pp. 533540, 2015.

[4] K. Kinjo, T. Matsui, T. Hisabe et al., "Increase in colonic diverticular hemorrhage and confounding factors," World Journal of Gastrointestinal Pharmacology and Therapeutics, vol. 7, no. 3, p. 440, 2016.

[5] Y. Nakagawa, T. Kamano, N. Ohmiya, T. Tahara, T. Shibata, and I. Hirata, "Effective management of severe diverticular bleeding in Japanese patients," Journal of Colitis \& Diverticulitis, vol. 1, no. 1, pp. 1-6, 2016.

[6] N. Nagata, N. Ishii, N. Manabe et al., "Guidelines for colonic diverticular bleeding and colonic diverticulitis: Japan gastroenterological association," Digestion, vol. 99, no. Suppl. 1, pp. 1-26, 2019.

[7] N. Ishii, T. Setoyama, G. A. Deshpande et al., "Endoscopic band ligation for colonic diverticular hemorrhage," Gastrointestinal Endoscopy, vol. 75, no. 2, pp. 382-387, 2012.

[8] D. Akutsu, T. Narasaka, K. Kobayashi et al., "Newly developed endoscopic detachable snare ligation therapy for colonic diverticular hemorrhage: a multicenter phase II trial (with videos)," Gastrointestinal Endoscopy, vol. 88, no. 2, pp. 370377, 2018.

[9] H. Doi, K. Sasajima, and M. Takahashi, "Using an over-thescope clip for colonic diverticular hemorrhage," Digestive Endoscopy, vol. 31, no. 3, pp. e80-e81, 2019.

[10] H. H. McGuire, "Bleeding colonic diverticula: a reappraisal of natural history and management," Annals of Surgery, vol. 220, no. 5, pp. 653-656, 1994.

[11] G. F. Longstreth, "Epidemiology and outcome of patients hospitalized with acute lower gastrointestinal hemorrhage: a population-based study," The American Journal of Gastroenterology, vol. 92, no. 3, pp. 419-424, 1997.

[12] D. M. Jensen, G. A. Machicado, R. Jutabha, and T. O. G. Kovacs, "Urgent colonoscopy for the diagnosis and treatment of severe diverticular hemorrhage," New England Journal of Medicine, vol. 342, no. 2, pp. 78-82, 2000.

[13] R. L. Smoot, C. J. Gostout, E. Rajan et al., "Is early colonoscopy after admission for acute diverticular bleeding needed?," The American Journal of Gastroenterology, vol. 98, no. 9, pp. 1996-1999, 2003.

[14] B. T. Green, D. C. Rockey, G. Portwood et al., "Urgent colonoscopy for evaluation and management of acute lower gastrointestinal hemorrhage: a randomized controlled trial," The American Journal of Gastroenterology, vol. 100, no. 11, pp. 2395-2402, 2005.
[15] L. Laine and A. Shah, "Randomized trial of urgent vs. elective colonoscopy in patients hospitalized with lower GI bleeding," American Journal of Gastroenterology, vol. 105, no. 12, pp. 2636-2641, 2010.

[16] T. Rustagi and T. R. McCarty, "Endoscopic management of diverticular bleeding," Gastroenterology Research and Practice, vol. 2014, Article ID 353508, 5 pages, 2014.

[17] N. Ishii, N. Hirata, F. Omata et al., "Location in the ascending colon is a predictor of refractory colonic diverticular hemorrhage after endoscopic clipping," Gastrointestinal Endoscopy, vol. 76, no. 6, pp. 1175-1181, 2012.

[18] A. Yamada, R. Niikura, S. Yoshida, Y. Hirata, and K. Koike, "Endoscopic management of colonic diverticular bleeding," Digestive Endoscopy, vol. 27, no. 7, pp. 721-726, 2015.

[19] R. Niikura, N. Nagata, A. Yamada et al., "Efficacy and safety of early vs elective colonoscopy for acute lower gastrointestinal bleeding," Gastroenterology, vol. 158, no. 1, pp. 168-175, 2020.

[20] M. A. Meyers, D. R. Alonso, G. F. Gray, and J. W. Baer, "Pathogenesis of bleeding colonic diverticulosis," Gastroenterology, vol. 71, no. 4, pp. 577-583, 1976.

[21] M. Lewis, "Bleeding colonic diverticula," Journal of Clinical Gastroenterology, vol. 42, no. 10, pp. 1156-1158, 2008.

[22] T. Obana, N. Fujita, R. Sugita et al., "Prospective evaluation of contrast-enhanced computed tomography for the detection of colonic diverticular bleeding," Digestive Diseases and Sciences, vol. 58, no. 7, pp. 1985-1990, 2013.

[23] N. Nagata, R. Niikura, T. Aoki et al., "Role of urgent contrastenhanced multidetector computed tomography for acute lower gastrointestinal bleeding in patients undergoing early colonoscopy," Journal of Gastroenterology, vol. 50, no. 12, pp. 1162-1172, 2015.

[24] C. J. Laing, T. Tobias, D. I. Rosenblum, W. L. Banker, L. Tseng, and S. W. Tamarkin, "Acute gastrointestinal bleeding: emerging role of multidetector CT angiography and review of current imaging techniques," Radiographics, vol. 27, no. 4, pp. 1055-1070, 2007.

[25] R. Loffroy, "Multidetector CT angiography for the detection of colonic diverticular bleeding: when, how, and why?" Digestive Diseases and Sciences, vol. 58, no. 7, pp. 1822-1824, 2013.

[26] N. Sengupta, E. B. Tapper, and J. D. Feuerstein, "Early versus delayed colonoscopy in hospitalized patients with lower gastrointestinal bleeding: a meta-analysis," Journal of Clinical Gastroenterology, vol. 51, no. 4, pp. 352-359, 2017.

[27] A. Seth, M. A. Khan, R. Nollan et al., "Does urgent colonoscopy improve outcomes in the management of lower gastrointestinal bleeding?" The American Journal of the Medical Sciences, vol. 353, no. 3, pp. 298-306, 2017.

[28] A. M. Kouanda, M. Somsouk, J. L. Sewell, and L. W. Day, "Urgent colonoscopy in patients with lower GI bleeding: a systematic review and meta-analysis," Gastrointestinal Endoscopy, vol. 86, no. 1, pp. 107-117, 2017.

[29] L. L. Strate and I. M. Gralnek, "ACG clinical guideline: management of patients with acute lower gastrointestinal bleeding," American Journal of Gastroenterology, vol. 111, no. 4, pp. 459-474, 2016.

[30] N. Ishii, F. Omata, N. Nagata, and M. Kaise, "Effectiveness of endoscopic treatments for colonic diverticular bleeding," Gastrointestinal Endoscopy, vol. 87, no. 1, pp. 58-66, 2018.

[31] T. Ikeya, N. Ishii, K. Nakano et al., "Risk factors for early rebleeding after endoscopic band ligation for colonic diverticular hemorrhage," Endoscopy International Open, vol. 03, no. 05, pp. E523-E528, 2015.

[32] T. Kaltenbach, R. Watson, J. Shah et al., "Colonoscopy with clipping is useful in the diagnosis and treatment of 
diverticular bleeding," Clinical Gastroenterology and Hepatology, vol. 10, no. 2, pp. 131-137, 2012.

[33] S. F. Pasha, A. Shergill, R. D. Acosta et al., "The role of endoscopy in the patient with lower GI bleeding," Gastrointestinal Endoscopy, vol. 79, no. 6, pp. 875-885, 2014.

[34] K. Nakano, N. Ishii, T. Ikeya et al., "Comparison of long-term outcomes between endoscopic band ligation and endoscopic clipping for colonic diverticular hemorrhage," Endoscopy International Open, vol. 03, no. 05, pp. E529-E533, 2015.

[35] N. Nagata, R. Niikura, T. Aoki et al., "Impact of discontinuing non-steroidal antiinflammatory drugs on long-term recurrence in colonic diverticular bleeding," World Journal of Gastroenterology, vol. 21, no. 4, pp. 1292-1298, 2015.

[36] M. Taki, T. Oshima, K. Tozawa et al., "Analysis of risk factors for colonic diverticular bleeding and recurrence," Medicine (Baltimore), vol. 96, no. 38, Article ID e8090, 2017.

[37] N. Nagata, R. Niikura, T. Sakurai et al., "Safety and effectiveness of early colonoscopy in management of acute lower gastrointestinal bleeding on the basis of propensity score matching analysis," Clinical Gastroenterology and Hepatology, vol. 14, no. 4, pp. 558-564, 2016. 


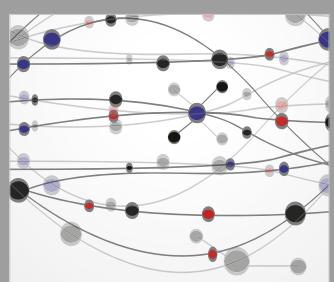

The Scientific World Journal
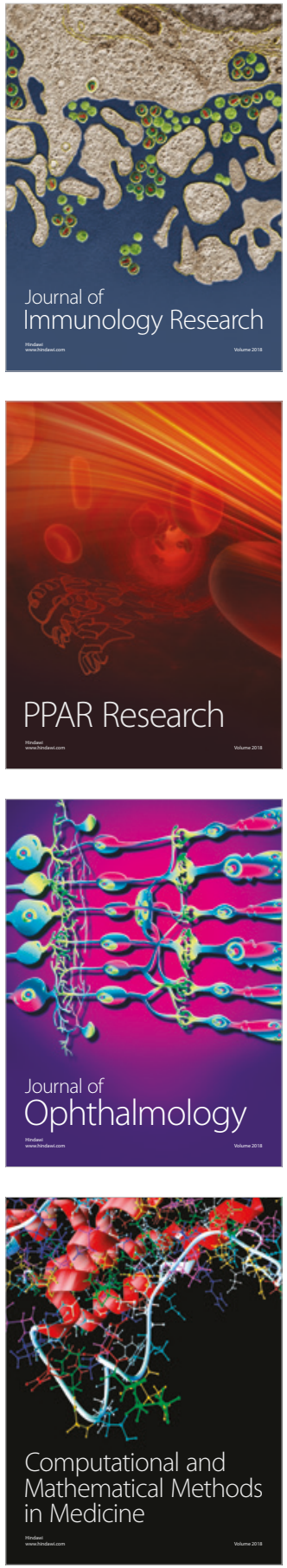

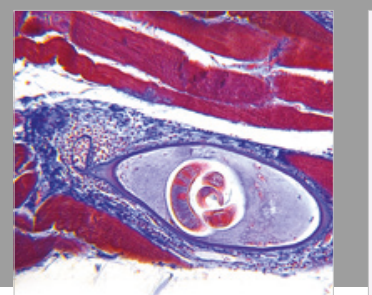

Gastroenterology Research and Practice

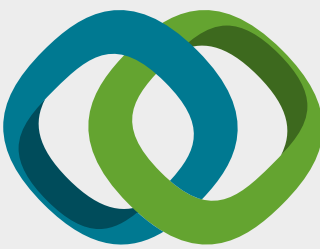

\section{Hindawi}

Submit your manuscripts at

www.hindawi.com
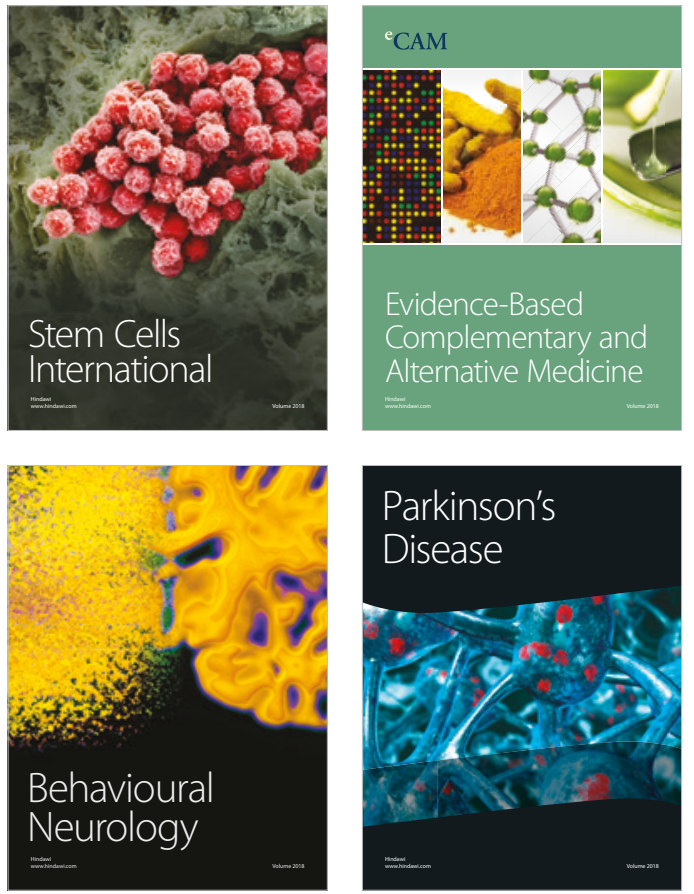

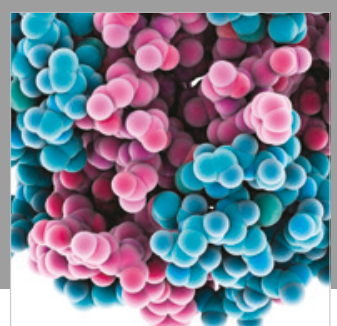

ournal of

Diabetes Research

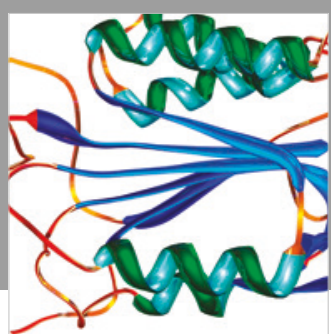

Disease Markers
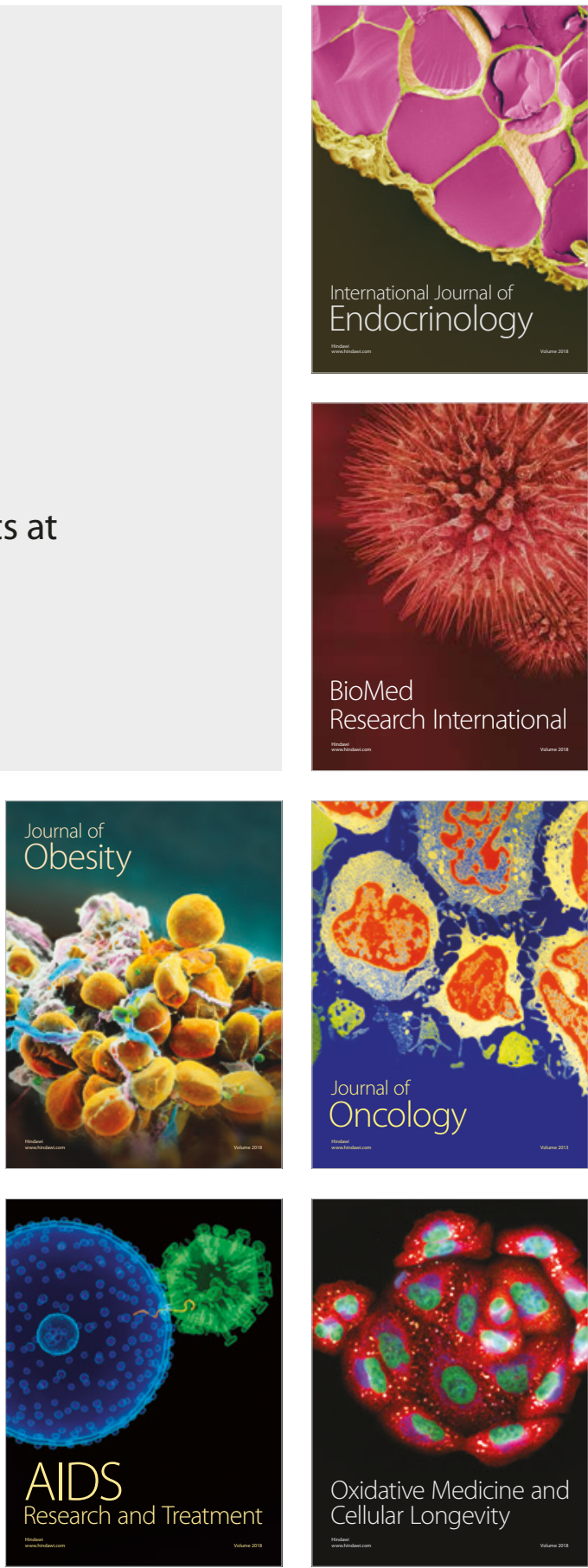